\title{
Tomonoid extensions: the key for the construction of t-norms
}

\author{
Thomas Vetterlein \\ Johannes Kepler University Linz, Altenberger Straße 69, 4040 Linz, Austria
}

\begin{abstract}
Triangular norms, or t-norms for short, play an important role for the semantics of fuzzy logics. Although an enormous number of examples and a remarkable number of construction methods for this kind of operation has been established, a uniform approach is still outstanding. This paper is devoted to a specific algebraic-geometrical framework within which t-norms, up to isomorphism, can be described systematically. By means of a detailed review of an example case, we show how the approach can be applied to the construction of left-continuous t-norms in a straightforward way.
\end{abstract}

Keywords: Triangular norms, totally ordered monoids

\section{Introduction}

Fuzzy logic is based on the idea to use a continuous set of truth degrees. Propositions are assigned values that vary continuously between 0 , representing "false", and 1, representing "true". Whereas the interpretation of truth degrees does not impose insurmountable problems, the mutual relationship of truth degrees on the one side and logical connectives on the other side has been a challenge. Let $\varphi$ and $\psi$ be propositions of a fuzzy logic. If $\varphi$ is assigned $s \in[0,1]$ and $\psi$ is assigned $t \in[0,1]$, with which value $u$ should we endow the conjunction of $\varphi$ and $\psi$ ? According to a further basic principle of fuzzy logic, $u$ is determined solely by $s$ and $t$. That is, there is a binary operation $\odot:[0,1] \times[0,1] \rightarrow[0,1]$, and $u=s \odot t$. Some basic assumptions commonly made for $\odot$ are not sufficient to single out a particular operation, but lead at least to the decision that $\odot$ should be a t-norm: associative, commutative, in both arguments isotone, and such that 1 is a neutral element.

For the basics of t-norm based many-valued logics, we refer to P. Hájek's seminal monograph [3]. The theory of t-norms has independently been developed into manifold directions. For an overview, see, e.g., [6]. T-norms can, first of all, be the topic of an investigation in the same style like two-place real functions in general; notions from real analysis are applicable. For the sake of a systematisation of t-norms, however, it is common to identify socalled isomorphic t-norms. Two t-norms $\odot_{1}$ and $\odot_{2}$ are isomorphic if there is an order automorphism $\iota:[0,1] \rightarrow[0,1]$ such that $a \odot_{2} b=\iota\left(\iota^{-1}(a) \odot_{1} \iota^{-1}(b)\right)$ for any $a, b \in[0,1]$. Accepting this identification, we are faced with the task to characterise t-norms by algebraic means.

It is not straightforward to see which type of algebraic structures is appropriate for our purposes. As a base set, we are given the real unit interval $[0,1]$; this is a complete totally ordered set. Endowed with the operation $\odot,[0,1]$ is a commutative monoid, where the neutral element is 1 . The order of $[0,1]$ and the monoidal structure are furthermore interrelated by the isotonicity assumption. We conclude that $[0,1]$, endowed with $\leq, \odot$, and 1 , is a totally ordered monoid, or tomonoid for short.

In a sense, tomonoids provide the minimal algebraic framework needed for t-norms. Here, we will not go beyond it. Tomonoids arising from t-norms are commutative, negative, and quantic. For the more general topic of commutative tomonoids, we refer to the comprehensive paper [2] and the references given there. We note that we could have chosen equally well complete MTL-algebras as our basic notion. The additional implication operation, however, is not of help in our analysis, and we use it only occasionally. In contrast, it was a deliberate decision not to make quantales our basic notion; our setting would in this case be less flexible when compared to tomonoids or MTL-algebras.

The probably most basic way of examining tomonoids is to study their quotients. Quotients of residuated lattices have been characterised in [1]; here, we use the analogous construction for tomonoids. Namely, each filter of a negative, commutative tomonoid gives rise to a quotient, and the set of all quotients obtained in this way forms a chain. If this chain is finite, we may say that the tomonoid is the result of step-wise construction process; in each step, the tomonoid is, as we say, extended to a more complex one. In fact, the crucial problem in our context is to characterise extensions: to describe in which way we may construct a tomonoid out of a given one such that the latter is a quotient of the former.

Although it seems to be unfeasible to determine all possible extensions of a tomonoid, there are approaches that help to make the problem accessible. There is, most important, a simple tool to represent the task in its full complexity: the Cayley transform. The idea to work with the set of inner translations of a tomonoid rather than with the 
tomonoid itself was already exploited in previous papers $[11,12]$. Whereas then our argumentation followed mainly a geometrical style, a recent work of ours paved the way for a purely algebraic view on the results in $[11,12]$; in [13], we have developed the theory of tomonoids with special regard to t-norm monoids. In particular, we observed that the Cayley transform represents in a clear way the filters of a tomonoid and the quotients induced by them. The results of [13] are well suited to bring some order into the diversity of t-norms and their construction methods. This task, however, is rather comprehensive and the topic of ongoing work.

The present paper is meant to demonstrate the effectiveness of the approach as regards the construction of left-continuous t-norms. To this end, we restrict to a particular interesting part of the theory, to a case where extensions of tomonoids are fully classifiable. The emphasis lies on keeping the construction process clear and transparent; several illustrations are included. We will proceed on the basis of an example that is complex enough to show the important aspects.

Our contribution is structured as follows. We introduce tomonoids and their representation by Cayley transforms in Section 2. In Section 3, we define quotients of tomonoids and explain how they are represented by means of Cayley transforms. As the key problem to be solved, we exhibit the extension of tomonoids, and we devote Section 4 to the analysis of a particular example. Namely, we explain the archimedean extension of a tomonoid that results in a t-norm, and we see that an extension is under the given circumstances fully classifiable. We conclude in Section 5 with an outlook to possible further research.

\section{Tomonoids}

Our topic are the following structures.

Definition 2.1 A structure $(\mathcal{L} ; \leq, \odot, 1)$ is a totally ordered monoid, or tomonoid for short, if, for all $a, b, c \in \mathcal{L},(\mathrm{i})(a \odot b) \odot c=a \odot(b \odot c)$, (ii) $a \odot 1=a$, and (iii) $a \leq b$ implies $a \odot c \leq b \odot c$ and $c \odot a \leq c \odot b$.

A tomonoid $\mathcal{L}$ is called commutative if $a \odot b=b \odot a$ for all $a, b \in \mathcal{L}$. $\mathcal{L}$ is called negative if $a \leq 1$ for all $a \in \mathcal{L}$. $\mathcal{L}$ is called quantic if (i) the total order $\leq$ is complete and (ii) for any elements $a, b_{\iota}, \iota \in I$, of $\mathcal{L}$ we have

$$
a \odot \bigvee_{\iota} b_{\iota}=\bigvee_{\iota}\left(a \odot b_{\iota}\right)
$$

T-norms [6] give rise to particular tomonoids. Let us endow the real unit interval $[0,1]$ with the natural order. Then a binary operation $\odot:[0,1] \times[0,1] \rightarrow$ $[0,1]$ is a t-norm if and only if $([0,1] ; \leq, \odot, 1)$ is a commutative, negative tomonoid.

In fuzzy logic, a t-norm $\odot$ is usually required to possess a residual implication, defined by $a \rightarrow b=$ $\max \{c \in[0,1]: a \odot c \leq b\}$ for $a, b \in[0,1]$. The implication exists exactly if $\odot$ is left-continuous. This fact motivates us to introduce for tomonoids an additional property, which is included in Definition 2.1 ; a t-norm $\odot$ is left-continuous if and only if the t-norm monoid based on $\odot$ is quantic. The notion "quantic" refers to the fact that a quantic tomonoid has the structure of a quantale [9]. We abbreviate in the sequel the properties "quantic, negative, and commutative" by "q.n.c.". Moreover, a q.n.c. tomonoid of the form $([0,1] ; \leq, \odot, 1)$, where $\odot$ is a left-continuous t-norm, is called a $t$-norm monoid.

According to Cayley's well-known representation theorem, any group can be identified with a transformation group. The idea is simple: the base set is the group itself, and with each element of the group we associate the mapping acting by right multiplication. The idea is applicable without change to the case of a semigroup. If the semigroup is actually a monoid, the neutral element is assigned the identity mapping. Finally, if a monoid is endowed with a translation-invariant total order, a further generalisation of the theorem is straightforward, as seen next.

In the sequel, we denote the identity function on a set $A$ by $i d_{A}$.

Proposition 2.2 Let $(\mathcal{L} ; \leq, \odot, 1)$ be a q.n.c. tomonoid. For each $a \in \mathcal{L}$, put

$$
\lambda_{a}: \mathcal{L} \rightarrow \mathcal{L}, \quad x \mapsto x \odot a,
$$

and let $\Lambda=\left\{\lambda_{a}: a \in \mathcal{L}\right\}$. Then $\Lambda$ is closed under the function composition $\circ$. Moreover, the pointwise order $\leq$ on $\Lambda$ is a complete total order; suprema are calculated pointwise; and $i_{\mathcal{L}}$ is the top element. Moreover,

$$
\iota: \mathcal{L} \rightarrow \Lambda, \quad a \mapsto \lambda_{a}
$$

is an isomorphism of the tomonoids $(\mathcal{L} ; \leq, \odot, 1)$ and $\left(\Lambda ; \leq, \circ, i d_{\mathcal{L}}\right)$.

Given a q.n.c. tomonoid $(\mathcal{L} ; \leq, \odot, 1)$, we will call the tomonoid $\left(\Lambda ; \leq, \circ, i d_{\mathcal{L}}\right)$ associated with $\mathcal{L}$ according to Proposition 2.2 the Cayley transform of $\mathcal{L}$. A Cayley transform can be characterised as follows [12]; cf. also [10]:

Proposition 2.3 Let $\left(\Lambda ; \leq, \circ, i d_{\mathcal{L}}\right)$ be the Cayley transform associated to $(\mathcal{L} ; \leq, \odot, 1)$. Then the following properties are fulfilled:

(F1) ○ is commutative.

(F2) For any $a \in \mathcal{L}$ there is a unique $\lambda \in \Lambda$ such that $\lambda(1)=a$.

(F3) For each $\lambda \in \Lambda$ and for any elements $a_{\iota} \in \mathcal{L}$, $\iota \in I$, we have $\lambda\left(\bigvee_{\iota} a_{\iota}\right)=\bigvee_{\iota} \lambda\left(a_{\iota}\right)$.

Conversely, let $\mathcal{L}$ be a complete totally ordered set, let 1 be the top element of $\mathcal{L}$, and let $\Lambda$ be a set of functions from $\mathcal{L}$ to $\mathcal{L}$ such that properties $(\mathrm{F} 1)$ (F3) hold. Then there is a unique binary operation $\odot$ on $\mathcal{L}$ such that $(\mathcal{L} ; \leq, \odot, 1)$ is a q.n.c. tomonoid 
and $\left(\Lambda ; \leq, \circ, \mathrm{id}_{\mathcal{L}}\right)$ is its associated Cayley transform. Namely,

$a \odot b=\lambda(b)$ where $\lambda \in \Lambda$ is such that $\lambda(1)=a$.

In particular, the left-continuous t-norms can be identified with sets of pairwise commuting, increasing, and left-continuous functions from $[0,1]$ to $[0,1]$ such that for any $a \in[0,1]$ exactly one of them maps 1 to $a$. Figure 1 shows an example.

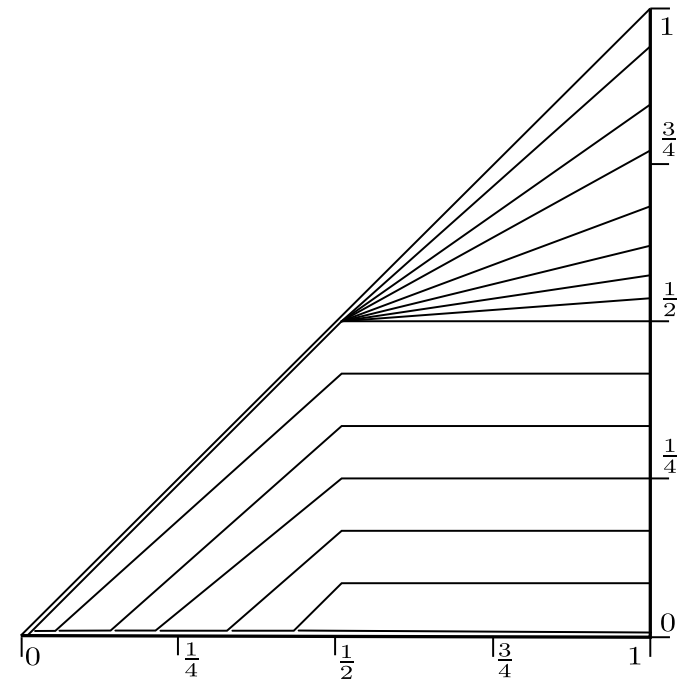

Figure 1: The Cayley transform of a t-norm, namely the ordinal sum of a Łukasiewicz t-norm and a product t-norm.

\section{Quotients of tomonoids}

To examine tomonoids and in particular of t-norm monoids, we follow common algebraic methods. We begin by defining congruences of tomonoids that are induced by filters.

Definition 3.1 Let $(\mathcal{L} ; \leq, \odot, 1)$ be a q.n.c. tomonoid. Then a filter of $\mathcal{L}$ is a subtomonoid $(F ; \leq, \odot, 1)$ of $\mathcal{L}$ such that $a \in F$ and $b \geq a$ imply $b \in F$.

Given a filter $F$ of $\mathcal{L}$, we define

$$
\begin{aligned}
a \sim_{F} b & \text { if } a=b, \\
& \text { or } a<b \text { and } b \odot c \leq a \text { for some } c \in F, \\
& \text { or } b<a \text { and } a \odot c \leq b \text { for some } c \in F
\end{aligned}
$$

for $a, b \in \mathcal{L}$, and we call $\sim_{F}$ the congruence induced by $F$.

Just like in the case of MTL-algebras [7], and analogously to the case of residuated lattices [1], filters induce in fact congruences. In the present context, there are actually more congruences available, a fact that we will, however, not exploit here.

Proposition 3.2 Let $(\mathcal{L} ; \leq, \odot, 1)$ be a q.n.c. tomonoid, and let $F$ be a filter of $\mathcal{L}$. Then each
$\sim_{F}$-class is an interval, that is, of the form $(a, b)$, $[a, b)$, or $(a, b]$ for some $a, b \in L$ such that $a<b$, or $[a, b]$ for some $a, b \in L$ such that $a \leq b$. We may define a total order on the quotient $\langle\mathcal{L}\rangle_{\sim_{F}}$ by putting

$$
\langle a\rangle_{\sim_{F}} \leq\langle b\rangle_{\sim_{F}} \text { if } a \sim_{F} b \text { or } a \leq b .
$$

Moreover, $\sim_{F}$ is a congruence of $\mathcal{L}$ as a monoid, hence we may define

$$
\langle a\rangle_{\sim_{F}} \odot\langle b\rangle_{\sim_{F}}=\langle a \odot b\rangle_{\sim_{F}} .
$$

Finally, $\left(\langle\mathcal{L}\rangle_{\sim_{F}} ; \leq, \odot,\langle 1\rangle_{\sim_{F}}\right)$ is a q.n.c. tomonoid.

Let $F$ be a filter of a q.n.c. tomonoid $\mathcal{L}$. We will write $\langle\mathcal{L}\rangle_{F}$ instead of $\langle\mathcal{L}\rangle_{\sim_{F}}$, and $\langle a\rangle_{F}$ instead of $\langle a\rangle_{\sim_{F}}$, where $a \in \mathcal{L}$. Consider the pair $\langle\mathcal{L}\rangle_{F}$ and $\mathcal{L}$. We will refer to $\langle\mathcal{L}\rangle_{F}$ as the tomonoid quotient of $\mathcal{L}$ by $F$; and conversely, we call $\mathcal{L}$ a tomonoid extension of $\langle\mathcal{L}\rangle_{F}$ by the extending tomonoid $F$.

Proposition 3.2 is straightforward apart from one point, which deserves a remark. Whereas it is immediate that the commutativity and negativity of a tomonoid is preserved by the formation of a quotient, the same is not so clear for the property of being quantic. This fact, for whose proof we refer to [14], reveals an inelegant aspect of our method. Congruences of quantic tomonoids are quantic again; but this does not imply that the natural homomorphism is a homomorphism of quantales. In fact, homomorphisms of the latter kind are required to preserve suprema; but in the present context the preservation of suprema is not guaranteed.

We next explain how the quotients of a t-norm monoid can be detected from its Cayley transform.

Definition 3.3 Let $\Lambda$ be the Cayley transform of a t-norm monoid based on the l.-c. t-norm $\odot$. Let $F \subseteq[0,1]$ be a filter of the t-norm monoid. Then we call

$$
\gamma_{F}:[0,1] \rightarrow[0,1], \quad x \mapsto \bigwedge_{a \in F} \lambda_{a}(x)
$$

the cut associated with $F$.

The cut $\gamma_{F}$ can be used to determine the $F$ classes as follows.

Proposition 3.4 Let $\odot$ be a l.-c. t-norm, and let $F$ be a filter. Then $\gamma_{F}$ is constant on each F-class. Indeed, we have

$$
\gamma_{F}(x)=\inf \langle x\rangle_{F}, \quad x \in[0,1]
$$

Conversely, let $R$ be a maximal interval on which $\gamma_{F}$ is constant. If $R$ is left-open, $R$ is an F-class. If $R$ possesses a smallest element $u$, either $\{u\}$ and $R \backslash\{u\}$ are $F$-classes or $R$ is an $F$-class, depending on whether or not there is an $x \in R \backslash\{u\}$ such that $\lambda_{f}(x)>u$ for any $f \in F$. 


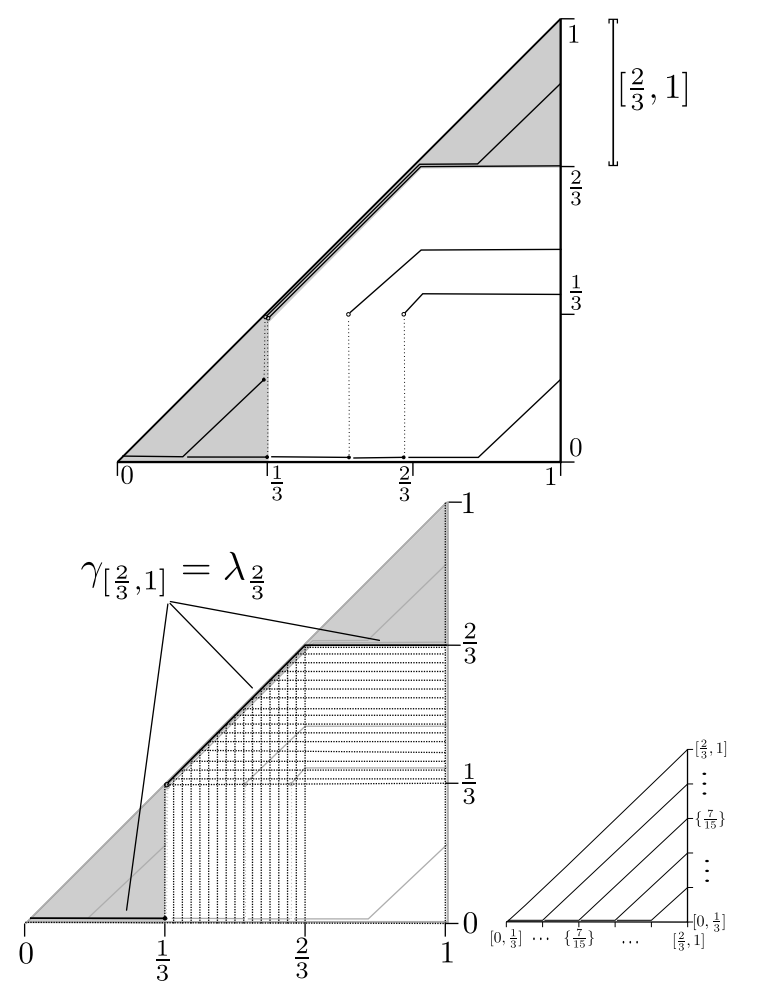

Figure 2: Above: The Cayley transform of the rotation-annihilation [5] of two Eukasiewicz tnorms. The filter $F=\left[\frac{2}{3}, 1\right]$ is highlighted in grey. Below left: Grid showing the quotient by $F$. Below right: The quotient by $F$.
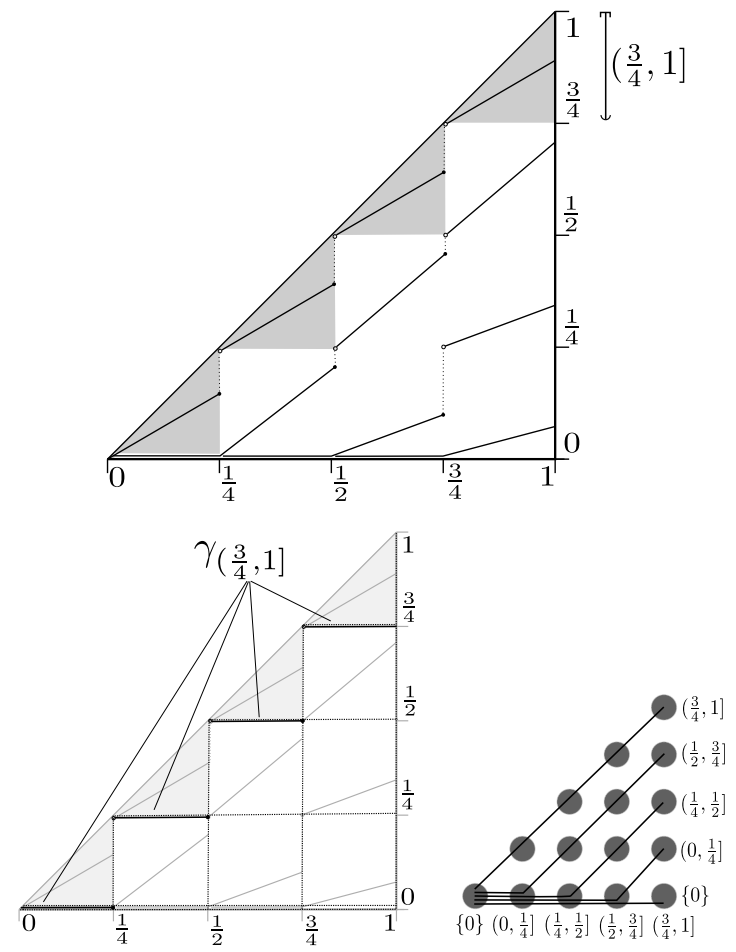

Figure 3: Above: The Cayley transform of a t-norm of Hájek [4]. The filter $F=\left(\frac{3}{4}, 1\right]$ is highlighted in grey. Below left: Grid showing the quotient by $F$. Below right: The quotient by $F$.
Let us interpret Proposition 3.4 in detail. Let a t-norm monoid be given and let $F$ be one of its filters. Then the quotient of the t-norm monoid induced by $F$ can be determined in the following way. We distinguish the case that $F$ possesses or does not possess a smallest element.

First case: The filter $F$ possesses the smallest element $d$; cf. Figure 2. Then $d$ is an idempotent, and $F=[d, 1]$. The cut associated with $F$ is $\gamma_{F}=\lambda_{d}$; in particular, $\gamma_{F}$ is contained in $\Lambda$. $\gamma_{F}$ is constant on closed intervals and has a step-like shape.

The transformations $\lambda_{f}$ such that $f \in F$ are all those that are above $\lambda_{d}$. Moreover, the congruence classes are exactly the intervals $[u, v]$, where $[u, v]$ is maximal such that $\lambda_{d}$ is constant on it.

Second case: The filter $F$ does not possess a smallest element; cf. Figure 3. Then $\gamma_{F}$ is not in $\Lambda$. Still, $\gamma_{F}$ has a step-like shape; however, the intervals on which $\gamma_{F}$ is constant might be left-open as well as right-open.

Although $\gamma_{F}$ is not in $\Lambda$, it is comparable with any element of $\Lambda$. The transformations $\lambda_{f}$ such that $f \in F$ are all those that are above $\gamma_{F}$. The congruence classes are determined as follows. Let $R \subseteq[0,1]$ be maximal such that $\gamma_{F}$ is constant on $R$; then $R$ is a singleton or of the form $(a, b),(a, b],[a, b)$, or $[a, b]$, where $a<b$. If $a \notin R$, then $R$ is a congruence class. If $a \in R$ and $R$ is not a singleton, the transformations $\lambda_{f}>\gamma_{F}$ need to be considered qualitatively. If at some point $x \in R$ such that $x \neq a$ there is no transformation mapping $x$ to $a, R \backslash\{a\}$ is a congruence class as well as the singleton $\{a\}$. Otherwise the whole $R$ is a congruence class.

We may draw a grid over the Cayley transform along the boundaries of the $F$-classes; cf. Figures 2 and 3. Associating with the transformations those squares that are traversed by them, we obtain the Cayley transform of the quotient by $F$.

\section{Extensions of tomonoids: a demonstration}

As we have seen, the determination of the quotient of a t-norm monoid induced by a filter is straightforward. The challenge of t-norm theory lies in the converse problem: how can we extend a given tomonoid to arrive at a new, more complex tomonoid, whose quotient is the one we started with? Loosely speaking, the Cayley transform of the given tomonoid needs to be refined; each transformation needs to be replaced by a bundle of new transformations acting on a base set that arises from the original base set by expanding each point to an interval.

In this section, we will, step by step, demonstrate on the basis of an example how the extension of 
a tomonoid can be performed. We will formulate in several propositions the hard facts, such that it will become clear to which extent we describe the general situation.

We first have to define the tomonoid $\mathcal{P}$ to be extended. We assume that $\mathcal{P}$ is four-element, $\mathcal{P}=$ $\{0,1,2,3\}$. Rather than providing a multiplication table for the monoidal operation of $\mathcal{P}$, we depict its Cayley transform. A Cayley transform is required to consist of pairwise commuting and increasing functions from $\mathcal{P}$ to $\mathcal{P}$ such that the top element 3 is mapped by exactly one transformation to each of the four elements. Our choice is seen in Figure 4, and it is immediate that any two of the four functions commute, hence we indeed define a tomonoid in this way.

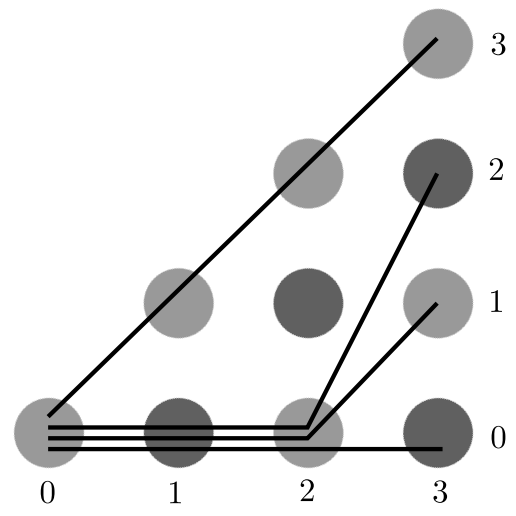

Figure 4: The Cayley transform of $\mathcal{P}$.

We wish to extend $\mathcal{P}$ by some tomonoid $F$, resulting in the tomonoid $\mathcal{L}$. Let us require that $\mathcal{L}$ is a t-norm monoid. This means that the new base set is $\mathcal{L}=[0,1]$, and the congruence classes are four intervals partitioning $[0,1]$. We assume the following partition:

$$
\left[0, \frac{1}{3}\right], \quad\left(\frac{1}{3}, \frac{2}{3}\right), \quad\left\{\frac{2}{3}\right\}, \quad\left(\frac{2}{3}, 1\right] .
$$

Figure 5 then shows the grid that results from $\mathcal{L}$ by forming the quotient according to the explanations of the previous section.

We now have to determine the actual translations of $\mathcal{L}$, that is, the elements of the Cayley transform of the t-norm monoid to be constructed. Loosely speaking, we have to replace the translations of $\mathcal{P}$ by bundles of translations of the new tomonoid. These translations apparently traverse triangular and square-shaped parts. This motivates the following tactics. We will not determine the translations as a whole, but "areawise". Namely, we will determine those parts of the translations that traverse a given triangle or a given square. We will "glue" them together afterwards.

We start with the topmost triangle, as seen in Figure 5, whose sides correspond to the top element of the quotient tomonoid, the interval $\left(\frac{2}{3}, 1\right]$. The following Proposition describes the general facts that

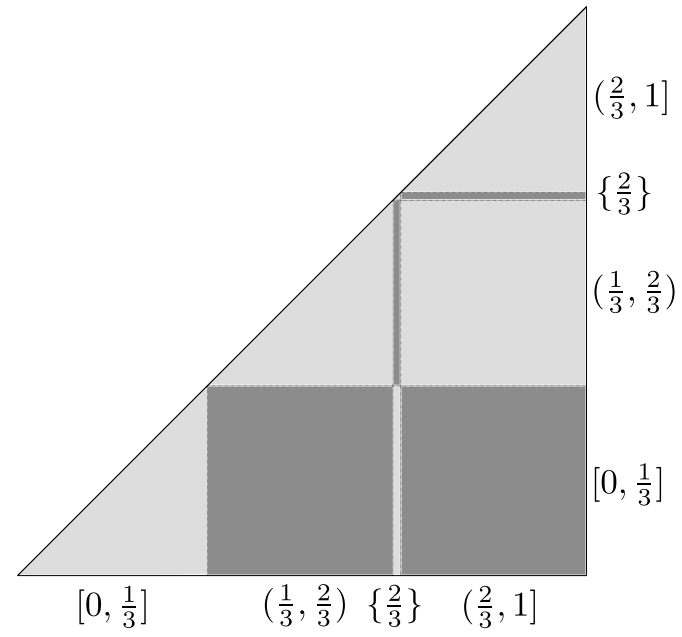

Figure 5: Grid of the tomonoid $\mathcal{L}$ belonging to the quotient by $F$. The colouring might help to identify this quotient with the Cayley transform shown in Figure 4.

apply here, where we use the following notation. Let $\mathcal{P}$ be a quotient of $\mathcal{L}$ by a filter $F$ of $\mathcal{L}$; we then always view the elements of $\mathcal{P}$ as subsets of $\mathcal{L}$. Furthermore, let $\lambda_{f}$ be a transformation of $\mathcal{L}$ such that $f \in F$. Then $\lambda_{f}$ maps $F$ to itself. We write $\lambda_{f}^{F}: F \rightarrow F$ for $\lambda_{f}$ with its domain and range being restricted to $F$, and we put $\Lambda^{F}=\left\{\lambda_{f}^{F}: f \in F\right\}$.

Proposition 4.1 Let $\mathcal{P}$ be the quotient of the q.n.c. tomonoid $(\mathcal{L} ; \leq, \odot, 1)$ by the filter $F$. Then the top element of $\mathcal{P}$ is $F$. Let $u=\inf F$; then $F$ is one of $(u, 1]$ or $[u, 1]$.

For each $f \in F, \lambda_{f}^{F}: F \rightarrow F, x \mapsto x \odot f$ is an increasing function whose right-limit at $u$ is $u$ and that maps 1 to $f$.

Moreover, the extending tomonoid $(F ; \leq, \odot, 1)$ is isomorphic to its associated Cayley transform $\Lambda^{F}=$ $\left\{\lambda_{f}^{F}: f \in F\right\}$, endowed with $\leq$, ०, and $\mathrm{id}_{F}$.

By Proposition 4.1, the topmost triangle simply represents the Cayley transform of the extending filter $F$. We have determined that $F=\left(\frac{2}{3}, 1\right]$, thus we have to choose a tomonoid with this base set.

The last assumption that we make is that our extension is, in a sense, minimal. Namely, we assume that the extending tomonoid $F$ is archimedean. A tomonoid $(L ; \leq, \odot, 1)$ is called archimedean if $a \leq$ $b<1$ implies $b^{n} \leq a$ for some $n$. Note that in this context archimedeanicity means that there are at most two archimedean classes. For the following theorem, see, e.g., [6].

In what follows, a real interval is meant to be a subset of $\mathbb{R}$ of the form $(a, b),[a, b),(a, b]$ for some $a<b$, or $[a, b]$ for some $a<b$.

Definition 4.2 (i) Let $\Phi$ contain the functions $\lambda_{t}:[0,1] \rightarrow[0,1], \quad x \mapsto(t+x-1) \vee 0$ for each $t \in[0,1]$. Then $\left(\Phi ; \leq, \circ, i d_{[0,1]}\right)$ is called the Eukasiewicz monoid. 
(ii) Let $\Phi$ contain the functions $\lambda_{t}:(0,1] \rightarrow$ $(0,1], x \mapsto t \cdot x$ for each $t \in(0,1]$. Then $\left(\Phi ; \leq, \circ, i_{(0,1]}\right)$ is called the product monoid.

Theorem 4.3 Let $(L ; \leq, \odot, 1)$ be a q.n.c. tomonoid. Let $L$ be archimedean, and assume that $L$ is a non-trivial real interval. If then $L$ possesses a bottom element, $L$ is isomorphic to the Lukasiewicz monoid; if $L$ does not possess a bottom element, $L$ is isomorphic to the product monoid.

Thus our extending filter $F$ must necessarily be isomorphic with the product monoid.

We now turn to the remaining triangles, each of which corresponds to an element of the quotient tomonoid $\mathcal{P}$. We generalise the notation needed for Proposition 4.4 as follows. Let $\lambda_{f}$ be a transformation of $\mathcal{L}$ such that $f \in F$, and let $R \in \mathcal{P}$ now be any $F$-class. Then $\lambda_{f}$ maps $R$ to $R$. We write $\lambda_{f}^{R}: R \rightarrow R$ for $\lambda_{f}$ with its domain and range being restricted to $R$, and we put $\Lambda^{R}=\left\{\lambda_{f}^{R}: f \in F\right\}$.

Proposition 4.4 Let $\mathcal{P}$ be the quotient of the q.n.c. tomonoid $(\mathcal{L} ; \leq, \odot, 1)$ by the filter $F$. Let $R \in \mathcal{P}$ be distinct from the top element and a non-singleton. Let $u=\inf R$ and $v=\sup R$; then $R$ is one of $(u, v)$, $[u, v),(u, v],[u, v]$. For each $f \in F, \lambda_{f}^{R}: R \rightarrow$ $R, \quad x \mapsto x \odot f$ is an increasing function whose right limit at $u$ is $u$.

$\Lambda^{R}=\left\{\lambda_{a}^{R}: a \in F\right\}$ is totally ordered w.r.t. the pointwise order, and arbitrary non-empty suprema exist and are calculated pointwise. The top element is $\mathrm{id}_{R}$.

Moreover, $\Lambda^{R}$ is closed under $\circ$, and

$$
F \rightarrow \Lambda^{R}, \quad a \mapsto \lambda_{a}^{R}
$$

is an epimorphism from $(F ; \leq, \odot, 1)$ to $\left(\Lambda^{R} ; \leq\right.$, $\left.\circ, i d_{R}\right)$.

Hence each triangle below the topmost one contains an algebra of commuting functions, which represents the extending filter $F$.

As we had decided that $F$ is archimedean, it will again follow that we do not have a choice. For the following theorem, see [11].

Definition 4.5 (i) Let $\Phi$ contain the functions $\lambda_{t}:[0,1) \rightarrow[0,1), \quad x \mapsto \frac{(t+x-1) \vee 0}{t}$ for each $t \in(0,1]$. Then $\left(\Phi ; \leq, \circ, \operatorname{id}_{[0,1)}\right)$ is called the reversed product monoid.

(ii) Let $\Phi$ contain the functions $\lambda_{t}:(0,1) \rightarrow$ $(0,1), x \mapsto x^{\frac{1}{t}}$ for each $t \in(0,1]$. Then $\left(\Phi ; \leq, \circ, i d_{(0,1)}\right)$ is called the power monoid.

Theorem 4.6 Let $\mathcal{P}$ be the quotient of a q.n.c. tomonoid $\mathcal{L}$ by an archimedean filter $F$ such that each element of $\mathcal{P}$ is order isomorphic to a real interval. Let $R \in \mathcal{P}$ be a non-singleton. Then $\Lambda^{R}$ is isomorphic to the power monoid, product monoid,

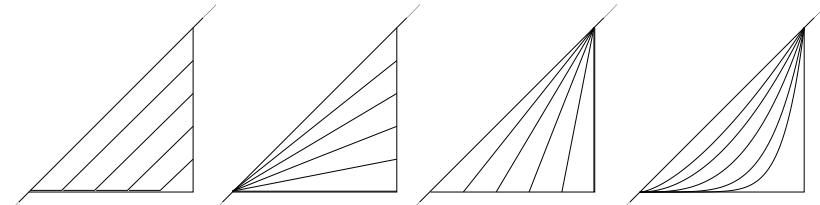

Figure 6: The Łukasiewicz, product, reversed product, and power monoid.

reversed product monoid, or Lukasiewicz monoid, depending on whether $R$ is of the form $(u, v),[u, v)$, $(u, v]$, or $[u, v]$ for some $u, v \in \mathcal{L}$ such that $u<v$.

Moreover, let $r, s \in R$ and $t \in F \backslash\{1\}$ such that $r \odot t=s$ and $s$ is not the smallest element of $R$. Given $F, \Lambda^{R}$, and the triple $(r, s, t)$, the assignment $F \rightarrow \Lambda^{R}, \quad f \mapsto \lambda_{f}^{R}$ is uniquely determined.

We conclude that our middle triangle, corresponding to the class $\left(\frac{1}{3}, \frac{2}{3}\right)$, is filled with a power monoid and the lowest triangle, corresponding to the class $\left[0, \frac{1}{3}\right]$, is filled with a Łukasiewicz monoid.

All functions we have determined so far are pieces of the translations by the extending filter $\left(\frac{2}{3}, 1\right]$. It remains to determine which piece of function belongs to which. By the second part of Theorem 4.6, we have to identify just one single translation that is not the identity.

We conclude that the construction of the translations by the extending filter is easy, and we note that the procedure is up to this point even in the general case feasible. Now comes the more critical part. However, under the assumptions we have madeintervals as congruence classes and archimedeanicity of the extending filter - the construction remains as easy as before.

What we can say about the general situation is contained in the next proposition. Once again, we need to introduce some notation, this time to be able to refer to what is inside the squares. Let $R, S, T \in \mathcal{P}$ be non-singletons and assume $R \odot T=$ $S$; assume further that $T$ is maximal with the property that multiplication with $R$ gives $S$, that is, $T=R \rightarrow S$. For $a \in T, \lambda_{a}$ maps $R$ to $S$. We write $\lambda_{a}^{R, S}: R \rightarrow S$ for $\lambda_{a}$ with its domain restricted to $R$ and its range restricted to $S$, and we put $\Lambda^{R, S}=\left\{\lambda_{t}^{R, S}: t \in T\right\}$.

Proposition 4.7 Let $\mathcal{P}$ be the quotient of the q.n.c. tomonoid $(\mathcal{L} ; \leq, \odot, 1)$ by the filter $F$. Let $R, S, T \in$ $\mathcal{P}$ be non-singletons and assume that $S=R \odot T$ and $T=R \rightarrow S$. Let $u=\inf R$ and $u^{\prime}=\inf S$. Then, for any $t \in T, \lambda_{t}^{R, S}: R \rightarrow S, \quad x \mapsto x \odot t$ is an increasing function whose right-limit at $u$ is $u^{\prime}$.

Moreover, $\Lambda^{R, S}=\left\{\lambda_{a}^{R, S}: a \in T\right\}$ is totally ordered w.r.t. the pointwise order, and the supremum of any non-empty bounded subset of $\Lambda^{R}$ exists and is calculated pointwise.

As mentioned above, our case allows a much stronger statement. 
Theorem 4.8 Let $\mathcal{P}$ be the quotient of a q.n.c. tomonoid $\mathcal{L}$ by an archimedean filter $F$ such that each element of $\mathcal{P}$ is order isomorphic to a real interval. Let $R, S, T \in \mathcal{P}$ be non-singletons and assume that $S=R \odot T$ and $T=R \rightarrow S$. Then either $S$ possesses a smallest element $u$ and $\Lambda^{R, S}$ contains only the constant u mapping. Or there is a unique set $\Xi$ of functions from $R$ to $S$, depending only on the functions $F \rightarrow \Lambda^{R}, f \mapsto \lambda_{f}^{R}$ and $F \rightarrow \Lambda^{S}, f \mapsto \lambda_{f}^{S}$, such that $\Lambda^{R, S}$ is either $\Xi$ or $\{f \in \Xi: f \leq h\}$ for some $h \in \Xi$.

Moreover, let $r \in R, s \in S$, and $t \in T$ be such that $r \odot t=s$ and $s$ is not the smallest element of $S$. Given $F, \Lambda^{R, S}$, the functions $F \rightarrow \Lambda^{R}, f \mapsto$ $\lambda_{f}^{R}$ and $F \rightarrow \Lambda^{S}, f \mapsto \lambda_{f}^{S}$, and the triple $(r, s, t)$, the assignment $T \rightarrow \Lambda^{R, S}, t \mapsto \lambda_{t}^{R, S}$ is uniquely determined.

According to Theorem 4.8, the "square-shaped parts" depend only on the "triangular parts" above and aside. We know that there are only four choices for the "triangles"; so there are at most 16 choices for the squares. Actually, there are less because not every combination works. Figure 7 provides a qualitative picture.
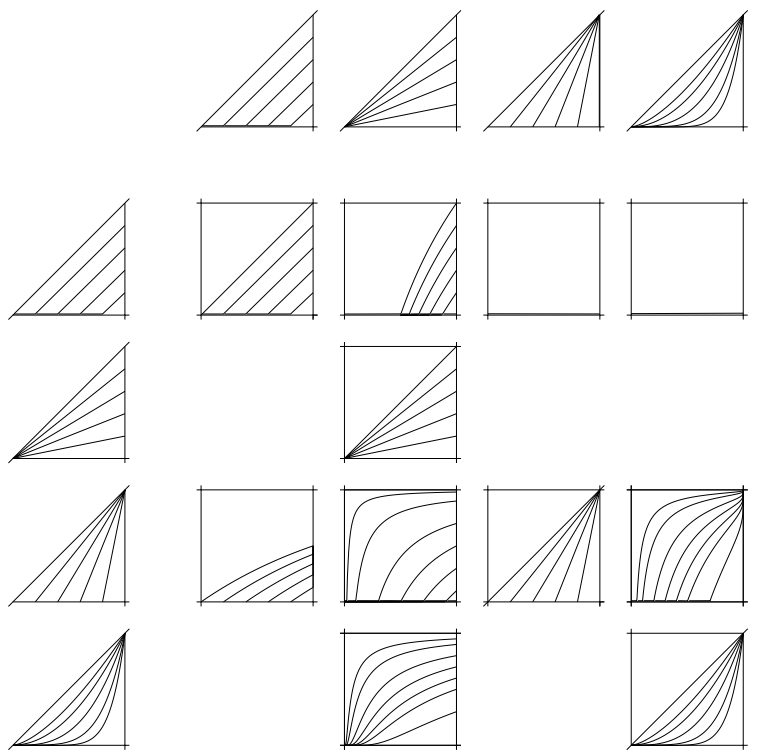

Figure 7: The possible translations restricted in domain and range to congruence classes. The picture is purely qualitative.

We finally conclude that our t-norm has the shape shown in Figure 8.

\section{Conclusion}

When viewed from an algebraic perspective, the theory of t-norms circles around the notion of a tomonoid extension. In fact, a t-norm gives rise to a tomonoid (i.e., totally ordered monoid); with each tomonoid, we may associate a chain of quotients; and so we may consider any tomonoid as the

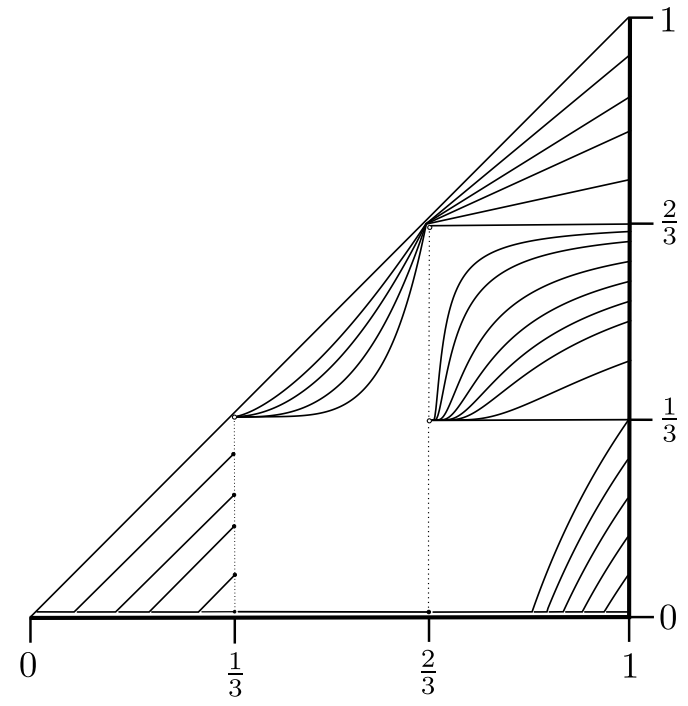

Figure 8: The finished t-norm.

final element of a linear construction, where from each point to any succeeding point the tomonoid is extended to a more complex one.

On the basis of an example, we have demonstrated that this construction process is under certain assumptions easily describable. We have considered the case that the extending tomonoid is archimedean and the congruence classes are order isomorphic to real intervals.

The general situation is certainly more complex. The chain of quotients need not be finite, not even countable, and in fact not even such that each element has a successor or predecessor. However, the continuous parts of this chain allow a description along the same lines as shown here. We then deal with extensions by semilattices, that is, tomonoids whose monoidal operation is simply the minimum.

For future research tackling the general case, it seems advisable to restrict to the finite case first. Already then, the problem is present in its full complexity. Many approaches might be possible; our suggestion is to consider the approach of M. Petrík and P. Sarkoci explained in [8]. Their idea is applicable to the case of general tomonoids and might allow a more transparent formulation of the extension problem than on the basis of Cayley transforms.

\section{References}

[1] K. Blount, C. Tsinakis, The structure of residuated lattices, Internat. J. Algebra Comput. 13:437-461, 2003.

[2] K. Evans, M. Konikoff, J. J. Madden, R. Mathis, G. Whipple, Totally ordered commutative monoids, Semigroup Forum 62:249-278, 2001.

[3] P. Hájek, "Metamathematics of Fuzzy Logic", Kluwer Acad. Publ., Dordrecht 1998. 
[4] P. Hájek, Observations on the monoidal tnorm logic, Fuzzy Sets Syst. 132:107-112, 2003.

[5] S. Jenei, Structure of left-continuous triangular norms with strong induced negations. II: Rotation-annihilation construction, J. Appl. Non-Class. Log. 11:351-366, 2001.

[6] E. P. Klement, R. Mesiar, E. Pap, "Triangular Norms", Kluwer Acad. Publ., Dordrecht 2000.

[7] C. Noguera, F. Esteva, J. Gispert, On some varieties of MTL-algebras, Log. J. IGPL 13:443-466, 2005.

[8] M. Petrík, P. Sarkoci, Associativity of triangular norms characterized by the geometry of their level sets, Fuzzy Sets Syst., to appear; online doi: 10.1016/j.fss.2012.01.008.

[9] K. I. Rosenthal, Quantales and their applications, Longman Scientific \& Technical, Essex 1990.

[10] S. Valentini, Representation theorems for quantales, Math. Logic Quart. 40:182-190, 1994.

[11] T. Vetterlein, Left-continuous t-norms as functional algebras, in: M. Štěpnička, V. Novák, U. Bodenhofer (eds.), "New Dimensions in Fuzzy Logic and Related Technologies", Proceedings of the 6th EUSFLAT conference (Ostrava 2007); 3743.

[12] T. Vetterlein, Regular left-continuous tnorms, Semigroup Forum 77:339-379, 2008.

[13] T. Vetterlein, The representation of finite positive commutative tomonoids by monoidal cones, submitted; available at http://www.flll.jku.at/sites/ default/files/u24/ endlicheTomonoide.pdf.

[14] T. Vetterlein, Totally ordered monoid based on triangular norms, submitted; available at http:// www.flll.jku.at/sites/default/ files/u24/tNorm-Zerlegung.pdf. 